\title{
Squalene epoxidase expression is associated with breast tumor progression and with a poor prognosis in breast cancer
}

\author{
NAH IHM KIM ${ }^{1}$, MIN HO PARK ${ }^{2}$, SUN-SEOG KWEON $^{3}$, NAMKI CHO $^{4}$ and JI SHIN LEE ${ }^{1}$ \\ Departments of ${ }^{1}$ Pathology, ${ }^{2}$ Surgery and ${ }^{3}$ Preventive Medicine, Chonnam National University Medical School, \\ Gwangju 61469; ${ }^{4}$ College of Pharmacy, Chonnam National University, Gwangju 61186, Republic of Korea
}

Received October 15, 2020; Accepted January 18, 2021

DOI: $10.3892 / \mathrm{ol} .2021 .12520$

\begin{abstract}
Differentially expressed genes (DEGs) have been previously identified using massive parallel RNA sequencing in matched normal, breast cancer (BC) and nodal metastatic tissues. Squalene epoxidase (SQLE), one of these DEGs, is a key enzyme in cholesterol synthesis. The aim of the present study was to investigate the potential involvement of SQLE in the tumorigenic process of $\mathrm{BC}$ and to determine its association with the clinical outcome of BC. SQLE mRNA expression was measured using reverse transcription-quantitative PCR in 10 pairs of ductal carcinoma in situ (DCIS) and BC tissues and their adjacent normal tissues. Immunohistochemical staining of SQLE on tissue microarray was performed in 26 normal breast, 79 DCIS and 198 BC samples. The role of SQLE as a prognostic biomarker in patients with $\mathrm{BC}$ has been verified using BreastMark. SQLE mRNA expression was significantly increased in DCIS and BC tissues compared with that in their adjacent normal tissues. High SQLE expression was detected in $0,48.1$ and $40.4 \%$ of normal breast, DCIS and BC tissues, respectively. SQLE expression in DCIS and BC tissues was significantly higher than that in normal breast tissues. High SQLE expression was observed in DCIS with higher nuclear grade, comedo-type necrosis and HER2 positivity. High SQLE expression in $\mathrm{BC}$ was associated with larger tumor size, nodal metastases, higher stage, HER2 subtype and distant metastatic relapse. High SQLE expression was associated with poor disease-free and overall survival, and independently predicted poor disease-free survival in patients with BC. Following BreastMark analysis, high SQLE mRNA expression in BC was significantly associated with a poor prognosis in the 'all', lymph node negative, lymph node positive, luminal A subtype and luminal B subtype groups. Therefore, SQLE expression may be upregulated during the tumorigenic process of $\mathrm{BC}$,
\end{abstract}

Correspondence to: Professor Ji Shin Lee, Department of Pathology, Chonnam National University Medical School, 160 Baekseo-ro, Dong, Gwangju 61469, Republic of Korea E-mail: jshinlee@hanmail.net

Key words: squalene epoxidase, breast, tumorigenesis, prognosis, immunohistochemistry and high SQLE expression may be a useful biomarker for predicting a poor prognosis in patients with BC.

\section{Introduction}

In Korean women, breast cancer (BC) is one of the most common type of cancer (1). Despite the significant improvements in the overall survival and quality of life for women with $\mathrm{BC}, \mathrm{BC}$ remains a leading cause of cancer-associated deaths (2). Therefore, it is necessary to develop new prognostic and therapeutic markers for patients with BC.

Cancer cells frequently exhibit altered cellular metabolism, which can mediate tumor progression and can be used for therapeutic purposes (3). Cholesterol is a unique lipid that is crucial for membrane formation, cell proliferation and cell differentiation (4). The critical role of cholesterol in the pathogenesis of various types of cancer, such as prostate and breast cancer, has been recognized in tumor cell proliferation, survival and treatment resistance (4-6). Moreover, several studies have revealed that inhibition of cholesterol synthesis at different steps results in human cancer cell death in both in vitro and in vivo models $(3,4,7)$. Overall, dysregulation of cholesterol metabolism may be a promising new therapeutic target for cancer treatment.

Squalene epoxidase (SQLE) is one of the rate-limiting enzymes in cholesterol synthesis by catalyzing the first step of squalene oxygenation (8). Previous studies have revealed that dysregulation of SQLE expression is involved in the molecular pathogenesis of various types of cancer, such as prostate cancer $(9,10)$, hepatocellular carcinoma (11), pancreatic cancer (12), esophageal squamous cell carcinoma (13) and squamous lung cancer (14). SQLE has been proposed as a new molecular marker to predict a poor prognosis in the aforementioned types of cancer (9-14).

Several studies have already been conducted with the aim of exploring the potential effect of the dysregulation of SQLE expression in BC (15-26). SQLE is involved in the maintenance of lipid droplet homeostasis in BC (15). Additionally, SQLE is involved in the process by which normal mammary fibroblasts induce a reversion of the malignant phenotype in primary BC (16). SQLE overexpression is more prevalent in groups with an unfavorable prognosis of stage I/II estrogen receptor-positive $\left(\mathrm{ER}^{+}\right) \mathrm{BC}(17)$, early-onset $\mathrm{BC}(18)$ and African-American patients with luminal A BC (19). Increased 
SQLE expression in patients with $\mathrm{ER}^{+} \mathrm{BC}$ has been associated with poor response to endocrine therapy (20). Furthermore, high SQLE expression has been associated with increased risk of BC recurrence (21-23). SQLE copy number amplification has been associated with its overexpression and poor prognosis (24). MYC gene amplification and aberrant SQLE methylation have also been observed in aggressive BC (25). Brown et al (26) have confirmed that SQLE is a true oncogene by clinically relevant amplification in BC. Brown et al (26) have demonstrated that SQLE overexpression represents an independent adverse prognostic factor and that SQLE inhibition may be a novel therapeutic target for BC. Most of the aforementioned SQLE studies in BC have been based on a molecular approach. Although ductal carcinoma in situ (DCIS) is a non-invasive cancerous lesion of the breast (27), to the best of our knowledge, there are no studies on the profile of SQLE expression during $\mathrm{BC}$ progression, including DCIS.

We have previously performed massive parallel RNA sequencing (RNA-Seq) analysis in 21 samples (normal, cancer and nodal metastases) from 7 patients with $\mathrm{ER}^{+}, \mathrm{HER} 2^{-} \mathrm{BC}$, revealing SQLE as one of the differentially expressed genes (DEGs) (28). Therefore, the present study evaluated the potential involvement of SQLE in the tumorigenic process of BC. Additionally, whether SQLE detection by immunohistochemistry may predict the prognosis in patients with $\mathrm{BC}$ was investigated. SQLE expression was examined in 10 pairs of DCIS and BC tissues and their adjacent normal tissues at the mRNA level by reverse transcription-quantitative PCR (RT-qPCR). In addition, immunohistochemical staining of SQLE expression on tissue microarray (TMA) was performed in 26 normal breast, 79 DCIS and 198 BC samples. The role of SQLE as a prognostic biomarker in patients with BC was then verified using BreastMark (29).

\section{Materials and methods}

Validation of SQLE RNA-Seq data by RT-qPCR. We have previously generated comprehensive gene expression profiles of matched normal, cancer and lymph node metastatic tissues from 7 patients with $\mathrm{ER}^{+}$, HER2- BC using RNA-Seq analysis (28). To validate the RNA-Seq data, SQLE mRNA expression was analyzed by RT-qPCR, as previously described (30). The isolated RNA used for RNA-Seq in our previous study (28) was used for RT-qPCR. The qPCR reaction was performed in a 7500 Fast Real-Time PCR System using TaqMan ${ }^{\circledR}$ Gene Expression Master Mix (Thermo Fisher Scientific, Inc.) using the following thermocycling conditions: Initial denaturation for $30 \mathrm{sec}$ at $95^{\circ} \mathrm{C}$, followed by 40 cycles at $95^{\circ} \mathrm{C}$ for $15 \mathrm{sec}$ and $60^{\circ} \mathrm{C}$ for $60 \mathrm{sec}$. The following probes (Thermo Fisher Scientific, Inc.) were used: Hs01123768_m1 (SQLE) and Hs02758991_g1 (GAPDH). The $2^{-\Delta \Delta \mathrm{Cq}}$ method (31) was used for data analysis and the value of $2^{-\Delta \Delta \mathrm{Cq}}$ indicated the fold change in SQLE expression normalized to GAPDH expression.

Samples for SQLE mRNA expression in DCIS and BC tissues and their adjacent normal tissues. New frozen samples of DCIS and BC tissues and their adjacent normal tissues $(\geq 2 \mathrm{~cm}$ from DCIS/BC tissues) were provided by the Biobank of Chonnam National University Hwasun Hospital (Jeollanam,
Republic of Korea), which is a member of the Korea Biobank Network, and were used for RT-qPCR, as aforementioned. The present study included 10 female patients each with DCIS or BC. The mean age in patients with DCIS was 57.2 years (median age, 57.6 years; age range, 48-73 years), while the mean age in patients with $\mathrm{BC}$ was 56.6 years (median age, 53.0 years; age range, $39-86$ years).

\section{SQLE expression in normal, DCIS and BC tissues}

Patients and tissues. Tissues were fixed in $10 \%$ formalin for $12-24 \mathrm{~h}$ at room temperature, cut into $<3$-mm-thick sections and embedded in paraffin. Formalin-fixed paraffin-embedded (FFPE) samples of normal breast tissues with no pathological lesions $(n=26)$, DCIS $(n=79)$ and BC $(n=198)$ were selected from female patients within Chonnam National University Hospital (Gwangju, Republic of Korea) and Chonnam National University Hwasun Hospital (Jeollanam, Republic of Korea). The mean age in patients with no pathological lesions was 44.3 years (median age, 45.5 years; age range, 25-69 years), the mean age in patients with DCIS was 49.3 years (median age, 48.0 years; age range, 27-73 years) and the mean age in patients with BC was 46.6 years (median age, 45.0 years; age range, 26-89 years). Patients diagnosed with BC between January 1997 and December 2002 were included in the present study and had a follow-up of $\geq 10$ years. After surgery, the patients underwent standard radiation therapy or adjuvant systemic therapy (hormone therapy or chemotherapy), according to the medical insurance program controlled by the Ministry of Health and Welfare of Korea. Medical records were reviewed to obtain clinicopathological information including patient outcome. ER, progesterone receptor (PR) and HER2 expression was assessed according to the American Society of Clinical Oncology/College of American Pathologists guidelines (32-34). DCIS samples between February 2005 and December 2011 were selected based on the availability of FFPE samples.

TMA construction. TMA blocks were constructed using one representative FFPE block in each case. Three cores of 1-mm diameter for $\mathrm{BC}$ and two cores of 2-mm diameter for normal breast and DCIS were punched from the donor block.

Immunohistochemistry and evaluation of immunostaining. SQLE expression was examined by immunohistochemical staining using the Bond-max automatic device (Leica Microsystems, Inc.), as previously described (35). Mouse polyclonal antibody against SQLE (1:200; cat. no. 042278; United States Biological) was used. According to a previous study (36), SQLE immunoreactivity was scored as 0 (negative), 1 (weak), 2 (moderate) or 3 (strong), based on the intensity of cytoplasmic staining. SQLE expression was evaluated by light microscopy (magnification, x200). Samples with intensity staining scores of 0-2 were considered as low SQLE expression, while scores of 3 were defined as high SQLE expression.

Validation of SQLE as a prognostic biomarker using BreastMark. SQLE was further analyzed to validate its prognostic value in patients with BC using the BreastMark database, as previously described (29).

Statistical analysis. The data of SQLE expression in matched normal, cancer and lymph node metastatic tissues from seven 

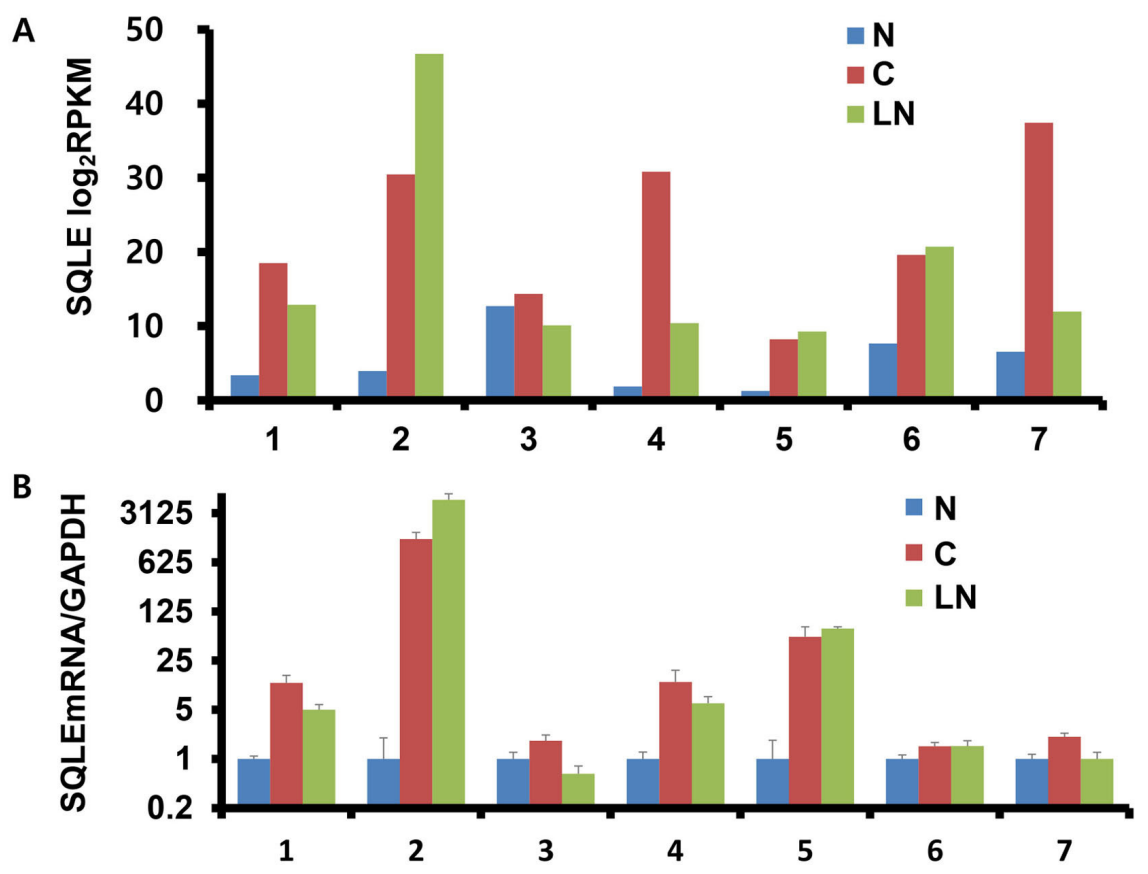

Figure 1. SQLE expression assessed via (A) RNA-Sequencing and (B) reverse transcription-quantitative PCR in matched N, C and LN tissues in seven patients with breast cancer. FPKM, fragments per kilobase of transcript per million mapped reads; N, normal; C, cancer; LN, lymph node metastatic; SQLE, squalene epoxidase.

patients were analyzed using one-way repeated measures ANOVA followed by post-hoc analysis with Fisher's Least Significant Difference adjustment for multiple comparisons. SQLE mRNA expression according to RT-qPCR in the DCIS and BC tissues and their adjacent normal tissues were compared using a paired Student's t-test (two-sided). Categorical nominal variables were tested using the $\chi^{2}$ test or Fisher's exact test. Linear-by-linear association was added to test for the trend. Univariate survival analysis was performed according to the Kaplan-Meier method and the differences in survival curves were assessed with the log-rank test. Cox's proportional hazard model was used for multivariate analysis. SPSS (v25 for windows; IBM Corp.) was used for all statistical analyses. $\mathrm{P}<0.05$ was considered to indicate a statistically significant difference.

\section{Results}

Validation of SQLE RNA-Seq data by RT-qPCR. SQLE expression was assessed by RNA-Seq (Fig. 1A) and RT-qPCR (Fig. 1B) in matched normal, cancer and nodal metastatic tissues in seven patients with BC. In the RNA-Seq results, SQLE expression (mean $\pm \mathrm{SD}$ ) was upregulated in BC tissues compared with in adjacent normal breast tissues $(22.8 \pm 10.4$ vs. $5.3 \pm 3.9$ for BC vs. normal, respectively; fold change, 3.915; $\mathrm{P}=0.007$ ) and subsequently unchanged when comparing nodal metastatic tissues with corresponding BC tissues (17.4 \pm 13.5 vs. $22.8 \pm 10.4$ for nodal vs. $\mathrm{BC}$; fold change, $-1.3014 ; \mathrm{P}=0.354$ ) (data not shown). For RT-qPCR, the unamplified total RNA (from the same batch used for RNA-Seq) was used as the template. Although no significant differences among groups were observed, SQLE expression was mainly upregulated in BC and nodal metastatic tissues compared with in adjacent normal breast tissues (data not shown). Overall, the results obtained with the two different techniques were consistent for $\sim 70 \%$ (5/7) of the samples tested.
SQLEmRNA in DCIS and BC tissues and their adjacent normal breast tissues. SQLE expression was assessed by RT-qPCR in 10 frozen DCIS tissues (Fig. 2A) and 10 BC tissues (Fig. 2B) and their respective adjacent normal breast tissues. The expression levels of SQLE mRNA (mean \pm SD) were significantly increased in DCIS and BC tissues compared with in their adjacent normal breast tissues $(6.27 \pm 7.54$ vs. $1.00 \pm 0.00$ for DCIS vs. normal, $\mathrm{P}<0.05 ; 14.02 \pm 22.95$ vs. $1.00 \pm 0.00$ for $\mathrm{BC}$ vs. normal, $\mathrm{P}<0.05$ ) (data not shown). No significant differences in SQLE mRNA expression (mean \pm SD) were observed between DCIS and BC (6.27 \pm 7.54 vs. 14.02 \pm 22.95 , respectively; $\mathrm{P}=0.323$ ) (data not shown).

SQLE expression in normal, DCIS and BC tissues. Normal breast tissues exhibited weak cytoplasmic SQLE expression (Fig. 3A and B). In DCIS and BC tissues, the carcinoma cells displayed variable SQLE expression (Fig. 3C-F). Table I summarizes SQLE expression in normal breast, DCIS and BC tissues. High SQLE expression was detected in 0/26 (0\%) of normal breast tissues, 38/79 (48.1\%) of DCIS samples, and 80/198 (40.4\%) of BC samples. Differential SQLE expression was noted in the DCIS and BC groups compared with in the normal breast group $(\mathrm{P}<0.05$, linear-by-linear association for trend). SQLE expression in DCIS and BC tissues was significantly higher compared with in normal breast tissues (DCIS vs. normal, 48.1 vs. $0 \%, \mathrm{P}<0.001$; BC vs. normal, 40.4 vs. $0 \%, \mathrm{P}<0.001)$. There was no significant difference in the expression levels of SQLE between DCIS and BC (DCIS vs. BC, 48.1 vs. $40.4 \%, \mathrm{P}=0.242$ ).

Tables II and III summarize the associations between SQLE expression and the clinicopathological features of patients with DCIS and BC, respectively. High SQLE expression in DCIS tissues was associated with nuclear grade $(\mathrm{P}<0.01)$, comedo-type necrosis $(\mathrm{P}<0.05)$ and HER2 

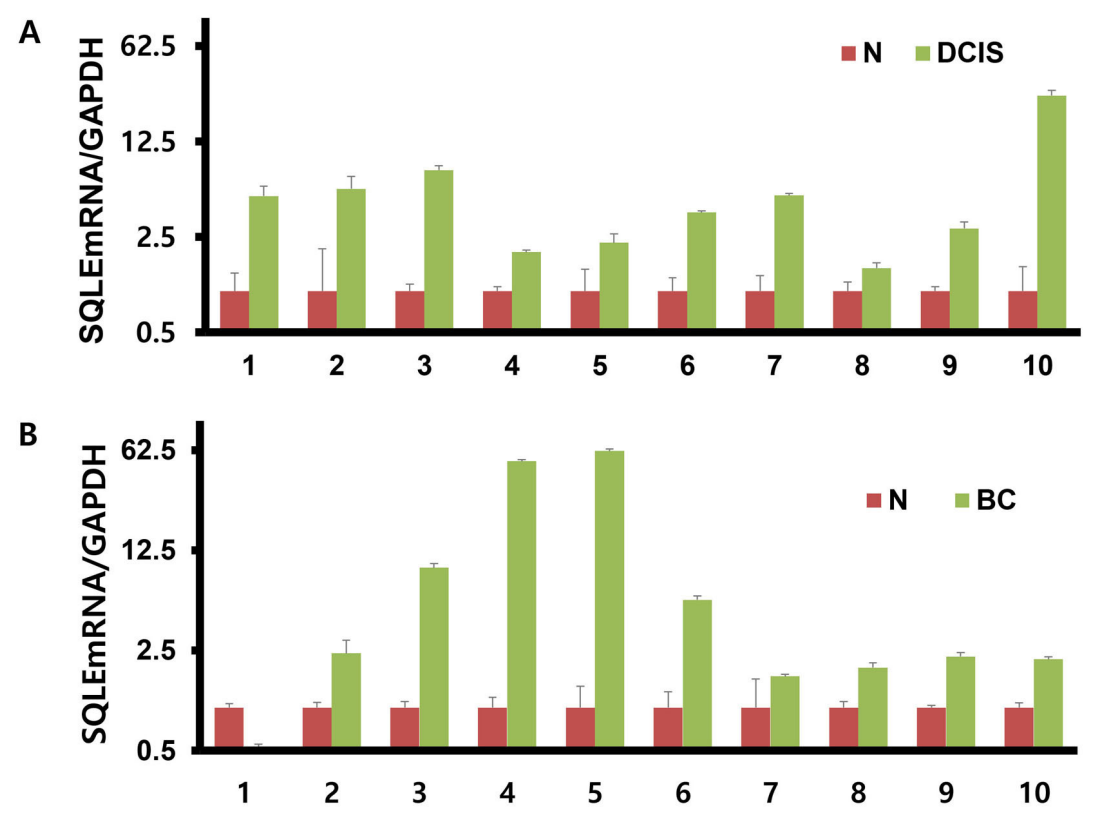

Figure 2. SQLE mRNA expression assessed via reverse transcription-quantitative PCR in (A) DCIS and (B) BC tissues and their adjacent N breast tissues. $\mathrm{N}$, normal; DCIS, ductal carcinoma in situ; BC, breast cancer; SQLE, squalene epoxidase.
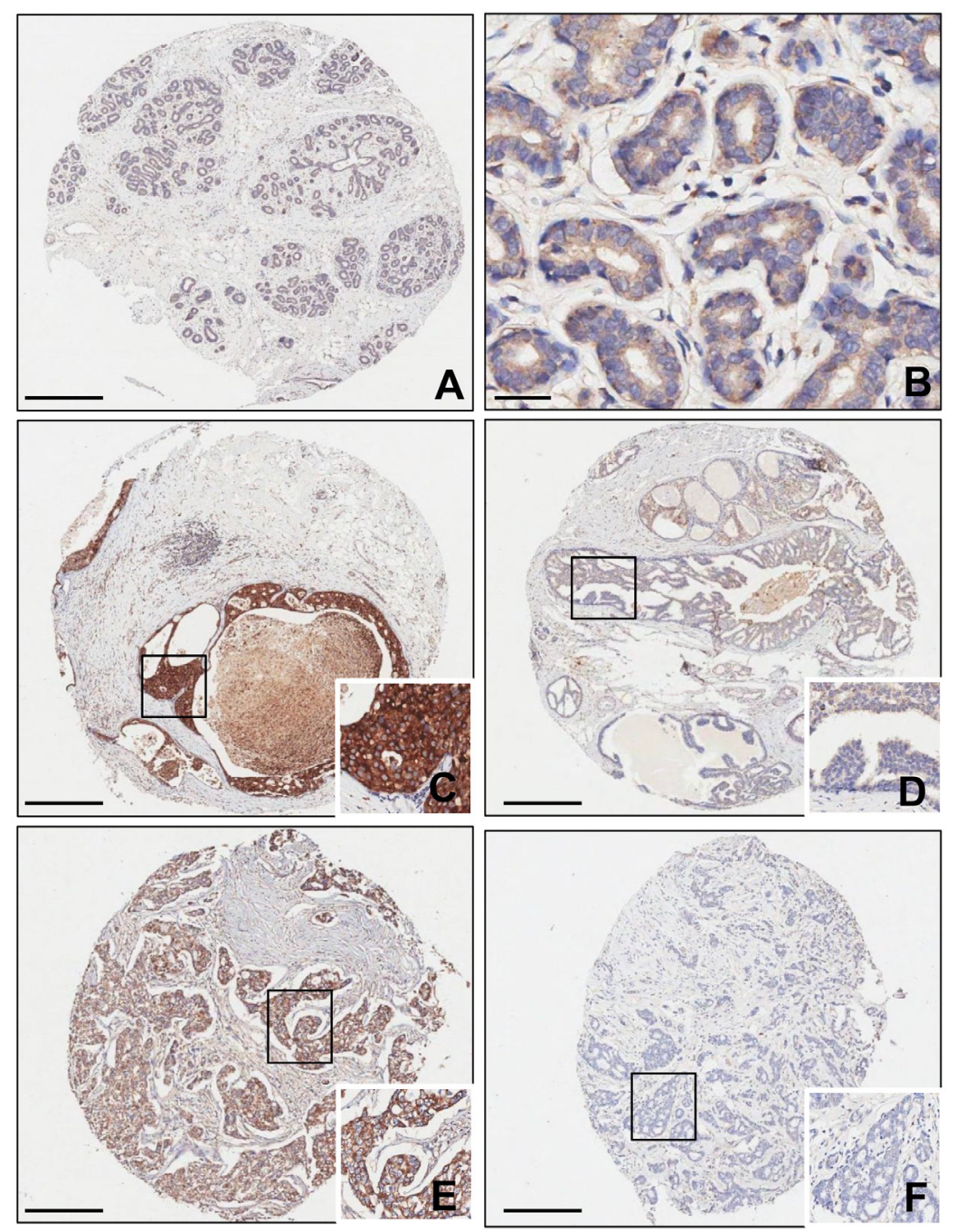

Figure 3. SQLE expression in normal breast, DCIS and BC tissues. (A and B) Normal breast tissue exhibited weak cytoplasmic SQLE expression (A: magnification, x4; scale bar, $500 \mu \mathrm{m}$; B: magnification, x400; scale bar, $60 \mu \mathrm{m}$ ). (C) High and (D) low SQLE expression in the cytoplasm of DCIS tissues. (E) High and (F) low SQLE expression in the cytoplasm of BC tissues. Magnification, $\mathrm{x} 4$; scale bar, $500 \mu \mathrm{m}$ (inlet magnification, $\mathrm{x} 400$ ). DCIS, ductal carcinoma in situ; $\mathrm{BC}$, breast cancer; SQLE, squalene epoxidase. 

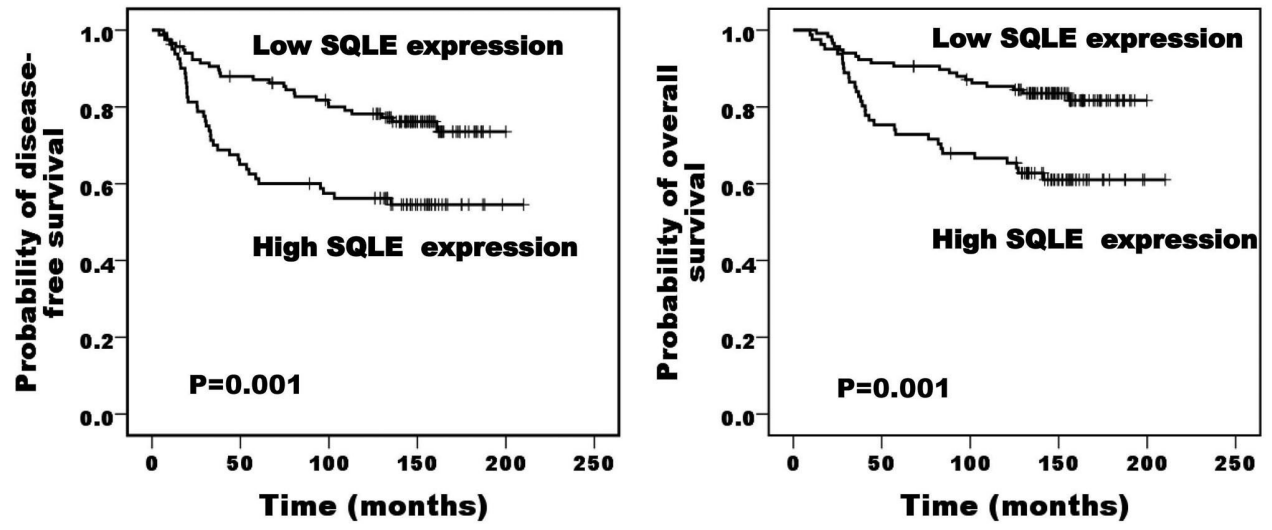

Figure 4. Survival of patients grouped according to SQLE expression. High SQLE expression predicted a significantly poor disease-free survival and overall survival ( $\mathrm{P}=0.001$ and $\mathrm{P}=0.001$, respectively). SQLE, squalene epoxidase.
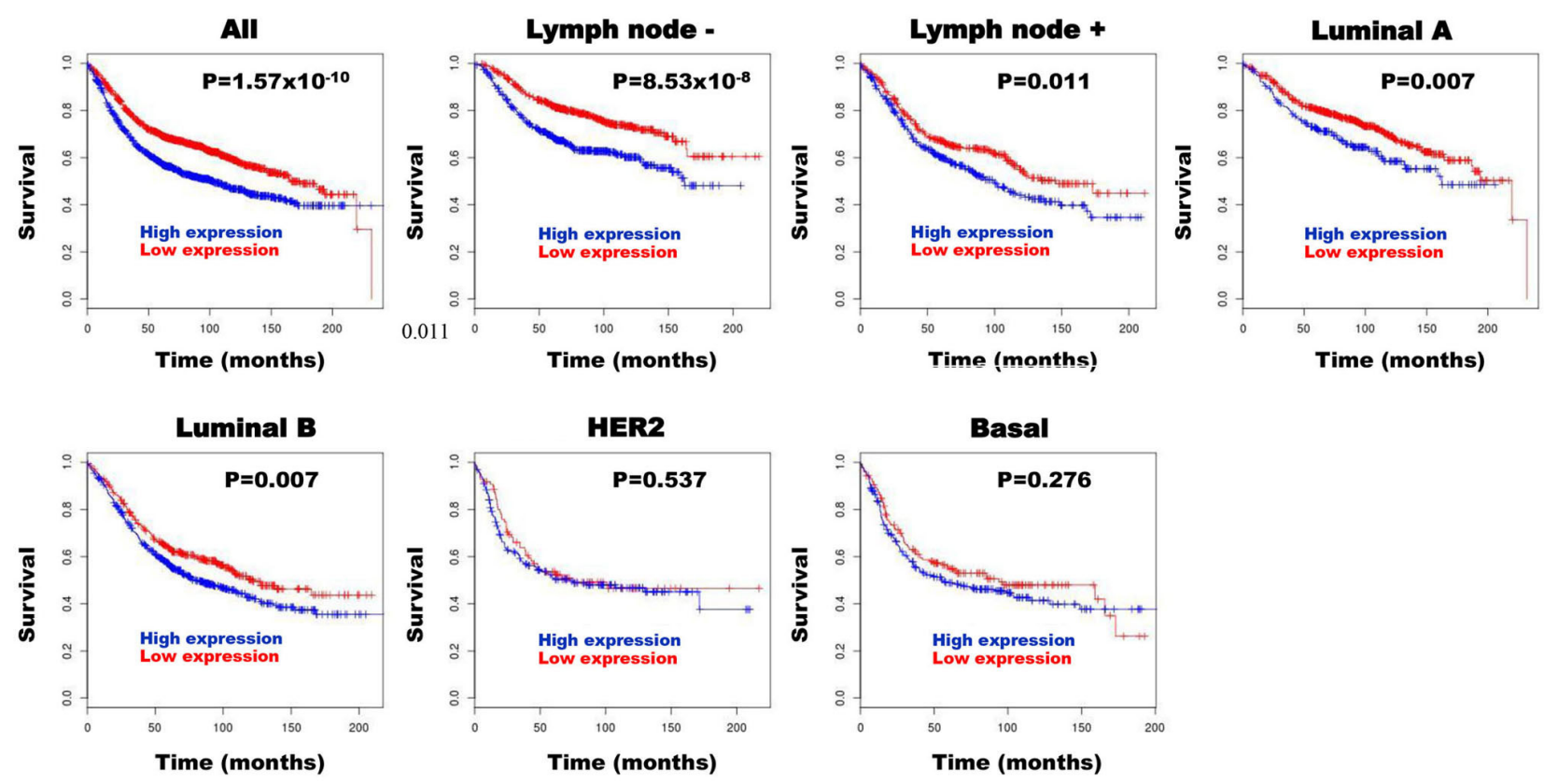

Figure 5. Prognostic role of squalene epoxidase expression in breast cancer assessed using BreastMark.

status $(\mathrm{P}<0.01)$ (Table II). Although not statistically significant, the percentage of high SQLE expression was higher in patients with recurrence $(5 / 7 ; 71.4 \%)$ than in patients without recurrence $(33 / 72 ; 45.8 \%)$ (Table II). High SQLE expression in $\mathrm{BC}$ tissues was associated with tumor size $(\mathrm{P}<0.05)$, nodal metastases $(\mathrm{P}<0.001)$, stage $(\mathrm{P}<0.01)$, molecular subtype $(\mathrm{P}<0.05)$ and distant metastatic relapse $(\mathrm{P}<0.05)$ (Table III).

Summary of survival analysis in patients with BC. Table IV summarizes the results of univariate survival analysis according to log-rank test for Kaplan-Meier survival analysis. Patients with high SQLE expression exhibited a poor prognosis for disease-free survival and overall survival compared with those with low expression ( $\mathrm{P}=0.001$ and $\mathrm{P}=0.001$, respectively; Fig. 4). Lymph node status and SQLE expression were independent poor prognostic factors for disease-free survival, while lymph node status was the only independent poor prognostic factor for overall survival (Table V).
Validation of SQLE as a prognostic biomarker using BreastMark. Following BreastMark analysis, high SQLE mRNA expression in patients with BC was significantly associated with poor prognosis in all groups [hazard ratio (HR), 1.467; $\left.\mathrm{P}=1.57 \times 10^{-10} ; \mathrm{n}=2,652\right)$, lymph node negative group (HR, 1.781; $\mathrm{P}=8.53 \times 10^{-8} ; \mathrm{n}=1,183$ ), lymph node positive group (HR, 1.337; $\mathrm{P}=0.011 ; \mathrm{n}=744$ ), luminal A subtype (HR, 1.427; $\mathrm{P}=0.007 ; \mathrm{n}=823)$ and luminal $\mathrm{B}$ subtype (HR, 1.284; $\mathrm{P}=0.007$; $\mathrm{n}=1,013$ ) (Fig. 5). However, high SQLE mRNA expression was not associated with prognosis in patients with HER subtype (HR, 1.117; $\mathrm{P}=0.537 ; \mathrm{n}=286)$ and basal subtype (HR, 1.169; $\mathrm{P}=0.276 ; \mathrm{n}=424)($ Fig. 5).

\section{Discussion}

SQLE expression is associated with pathogenesis and clinical outcome in various types of cancer (9-14). The present study demonstrated the upregulation of SQLE mRNA expression in DCIS and BC tissues compared with in their adjacent normal 
Table I. SQLE expression in normal breast, ductal carcinoma in situ and breast cancer tissues.

\begin{tabular}{lcc}
\hline Type of tissue & $\begin{array}{c}\text { High SQLE expression, } \\
\text { n/total n (\%) }\end{array}$ & P-value \\
\hline Normal & $0 / 26(0.0)$ & $0.018^{\mathrm{a}}$ \\
Ductal carcinoma in situ & $38 / 79(48.1)$ & \\
Breast cancer & $80 / 198(40.4)$ & \\
\hline a & \\
Pinear-by-linear association. Normal vs. ductal carcinoma in situ, \\
in situ vs. breast cancer, $\mathrm{P}=0.242$. SQLE, squalene epoxidase.
\end{tabular}

Table II. Association between SQLE expression and clinicopathological parameters of patients with ductal carcinoma in situ $(\mathrm{n}=79)$.

\begin{tabular}{|c|c|c|}
\hline Characteristics & $\begin{array}{l}\text { High SQLE } \\
\text { expression, } \\
\text { n/total n }(\%)\end{array}$ & P-value ${ }^{a}$ \\
\hline Age, years & & $>0.05$ \\
\hline$\leq 50$ & $21 / 45(46.7)$ & \\
\hline$>50$ & $17 / 34(50.0)$ & \\
\hline Size, cm & & $>0.05$ \\
\hline$\leq 2.5$ & $21 / 45(46.7)$ & \\
\hline$>2.5$ & $17 / 34(50.0)$ & \\
\hline Nuclear grade & & $<0.01$ \\
\hline 1 & 1/7 (14.3) & \\
\hline 2 & $19 / 46(41.3)$ & \\
\hline 3 & $18 / 26(69.2)$ & \\
\hline Comedo-type necrosis & & $<0.05$ \\
\hline No & $11 / 32(34.4)$ & \\
\hline Yes & $27 / 47(57.4)$ & \\
\hline Estrogen receptor- $\alpha$ & & $>0.05$ \\
\hline Negative & $17 / 28(60.7)$ & \\
\hline Positive & $21 / 51(41.2)$ & \\
\hline HER2 & & $<0.01$ \\
\hline Negative & $18 / 51(35.3)$ & \\
\hline Positive & 20/28 (71.4) & \\
\hline Recurrence & & $>0.05^{\mathrm{b}}$ \\
\hline No & $33 / 72(45.8)$ & \\
\hline Yes & 5/7 (71.4) & \\
\hline
\end{tabular}

${ }^{\mathrm{a}}$ Analyzed by $\chi^{2}$ test; ${ }^{\mathrm{b}}$ Analyzed by Fisher's exact test. SQLE, squalene epoxidase.

breast tissues. SQLE expression was associated with breast tumor progression and with a poor clinical outcome in patients with BC.

Cholesterol is the main sterol that is synthesized in all animal cells and is an essential structural component of cell membrane (4). An association between cholesterol and cancer has been previously recognized (6). Moreover, previous studies
Table III. Association between SQLE expression and clinicopathological parameters of patients with breast cancer $(n=198)$.

\begin{tabular}{|c|c|c|}
\hline Characteristics & $\begin{array}{l}\text { High SQLE } \\
\text { expression, } \\
\text { n/total n (\%) }\end{array}$ & $\mathrm{P}$-value ${ }^{\mathrm{a}}$ \\
\hline Age, years & & $>0.05$ \\
\hline$\leq 46$ & $40 / 112(35.7)$ & \\
\hline$>46$ & $41 / 86(47.7)$ & \\
\hline Histopathologic type & & $>0.05$ \\
\hline Invasive ductal carcinoma, NOS & 67/171 (39.2) & \\
\hline Invasive lobular carcinoma & $14 / 25(56.0)$ & \\
\hline Mucinous carcinoma & $0 / 2(0.0)$ & \\
\hline Tumor size, cm & & $<0.05$ \\
\hline$\leq 2$ & $11 / 35(31.4)$ & \\
\hline $2-5$ & $51 / 133(38.3)$ & \\
\hline$>5$ & $19 / 30(63.3)$ & \\
\hline Number of nodal metastasis & & $<0.001$ \\
\hline 0 & 29/105 (27.6) & \\
\hline $1-3$ & 25/51 (49.0) & \\
\hline $4-9$ & $13 / 25(52.0)$ & \\
\hline$\geq 10$ & $14 / 17(82.4)$ & \\
\hline Histological grade & & $>0.05$ \\
\hline 1 & $4 / 26(15.4)$ & \\
\hline 2 & 47/101 (46.5) & \\
\hline 3 & $30 / 71(42.3)$ & \\
\hline Stage & & $<0.01$ \\
\hline I & $11 / 35(31.4)$ & \\
\hline II & $37 / 112(33.0)$ & \\
\hline III & $33 / 51(64.7)$ & \\
\hline Estrogen receptor- $\alpha$ & & $>0.05$ \\
\hline Negative & $40 / 87(46.0)$ & \\
\hline Positive & $41 / 111(36.9)$ & \\
\hline Progesterone receptor & & $>0.05$ \\
\hline Negative & $39 / 87(44.8)$ & \\
\hline Positive & 42/111 (37.8) & \\
\hline HER2 & & $>0.05$ \\
\hline Negative & $64 / 162(39.5)$ & \\
\hline Positive & $17 / 36(47.2)$ & \\
\hline Molecular subtypes & & $<0.05$ \\
\hline Luminal & $50 / 135(37.0)$ & \\
\hline HER2 & $14 / 21(66.7)$ & \\
\hline Triple negative & $17 / 42(40.5)$ & \\
\hline Distant metastatic relapse & & $<0.05$ \\
\hline No & $52 / 146(35.6)$ & \\
\hline Yes & $29 / 52(55.8)$ & \\
\hline
\end{tabular}

${ }^{a}$ Analyzed by $\chi^{2}$ test. NOS, not otherwise specified; SQLE, squalene epoxidase.

have indicated that inhibition at different stages of cholesterol synthesis contributes to inhibition of tumor cell proliferation, cell death and resistance to therapies in cancer $(3,4,7)$. Overall, 
Table IV. Univariate analysis of prognostic factors in patients with breast cancer.

\begin{tabular}{lrr}
\hline Characteristics & Disease-free survival (P-value) & Overall survival (P-value) \\
\hline Age ( 46 vs. >46 years) & 0.972 & 0.522 \\
Histological type (invasive carcinoma of no special & 0.076 & 0.409 \\
type vs. other types) & & $<0.001$ \\
Tumor size (pT1 vs. pT2 vs. pT3) & $<0.001$ & $<0.001$ \\
Lymph node status (pN0 vs. pN1 vs. pN2 vs. pN3) & $<0.001$ & 0.105 \\
Histological grade (1 vs. 2 vs. 3) & 0.513 & $<0.001$ \\
Stage (I vs. II vs. III vs. IV) & $<0.001$ & 0.295 \\
Hormonal therapy (no vs. yes) & 0.395 & 0.017 \\
Chemotherapy/radiotherapy (no vs. yes) & 0.026 & 0.424 \\
Estrogen receptor- $\alpha$ status (negative vs. positive) & 0.430 & 0.625 \\
Progesterone receptor status (negative vs. positive) & 0.527 & 0.627 \\
HER-2 status (negative vs. positive) & 0.966 & 0.001
\end{tabular}

Table V. Multivariate analysis with Cox's proportional hazard model for prognostic factors in patients with breast cancer.

\begin{tabular}{|c|c|c|c|c|c|c|}
\hline \multirow[b]{2}{*}{ Characteristics } & \multicolumn{3}{|c|}{ Disease-free survival } & \multicolumn{3}{|c|}{ Overall survival } \\
\hline & HR & $95 \% \mathrm{CI}$ & P-value & HR & $95 \% \mathrm{CI}$ & P-value \\
\hline Age ( $\leq 46$ vs. $>46$ years $)$ & 0.072 & $0.566-1.539$ & 0.788 & 0.038 & $0.597-1.877$ & 0.845 \\
\hline Tumor size $(\leq 5$ vs. $>5 \mathrm{~cm})$ & 2.885 & $0.291-1.092$ & 0.089 & 1.492 & $0.320-1.303$ & 0.222 \\
\hline Lymph node status (negative vs. positive) & 6.443 & $0.212-0.819$ & 0.011 & 4.922 & $0.170-0.896$ & 0.027 \\
\hline Stage (I/II vs. III) & 0.440 & $0.394-1.584$ & 0.507 & 1.885 & $0.265-1.263$ & 0.170 \\
\hline Chemotherapy/radiotherapy (no vs. yes) & 1.285 & $0.193-1.551$ & 0.257 & 1.484 & $0.096-1.730$ & 0.223 \\
\hline Squalene epoxidase expression (low vs. high) & 4.079 & $0.354-0.985$ & 0.043 & 3.561 & $0.318-1.022$ & 0.059 \\
\hline
\end{tabular}

HR, hazard ratio.

dysregulation of cholesterol metabolism may be a new therapeutic target in cancer treatment.

SQLE encodes squalene epoxidase, one of the major enzymes in the late stages of cholesterol synthesis (8). SQLE catalyzes the oxidation of squalene to 2,3-oxidosqualene (squalene epoxide), and its inhibition can affect the synthesis of sterols and of the cell membrane, or even cell growth (3). Previous studies have indicated that dysregulation of SQLE is involved in the molecular pathogenesis of various types of cancer and has also been associated with poor prognosis (9-14). SQLE has been associated with radiation resistance in pancreatic cancer (12) and metastasis in esophageal squamous cell carcinoma (13), as well as with poor outcomes in patients with prostate cancer $(9,10)$, hepatocellular carcinoma (11) and squamous lung cancer (14).

Several studies have already been conducted to explore the potential role of the dysregulation of SQLE in BC (15-26). Polycarpou-Schwarz et al (16) identified a new microprotein gene, Cancer-Associated Small Integral Membrane Open reading frame 1 (CASIMO1), utilizing a microarray approach. CASIMO1 was found to be overexpressed and important for proper proliferation of breast cancer cells, and exerted its effects through interactions with SQLE maintaining lipid droplet homeostasis (16). Römer et al (15) assessed the ability of normal human mammary fibroblasts (HMFs) to induce reversion of the malignant phenotype of primary breast carcinoma cells in a three-dimensional cell culture model. The reversion of the malignant phenotype was detected in 5/13 primary breast carcinoma cell co-cultures with HMFs (15). Gene expression analysis revealed that SQLE expression was downregulated in the reverted cases compared with in the non-reverted cases (15). These findings were consistent with the role of SQLE in regulating BC progression and inhibition of differentiation (15). cDNA microarrays reported that SQLE was differentially expressed in BC and that SQLE expression was increased dramatically in cancer tissues compared with in normal and cancer-adjacent normal tissues $(18,19)$. In the present study, SQLE expression was directly compared in sets of matched normal breast, BC and nodal metastatic tissues using RNA-Seq and RT-qPCR. In the RNA-Seq data, SQLE expression was upregulated in BC tissues compared with in matched normal breast tissues, and was unchanged when comparing nodal metastatic tissues with matched BC tissues. Based on changes in gene expression during progression from normal tissue to cancer tissue and then to metastatic node of BC, SQLE may belong to genes 
associated with tumorigenesis (normal-cancer transition) rather than metastasis (cancer-metastasis transition).

Although BC is believed to develop from DCIS (27), to the best of our knowledge, there are no studies that have examined the association between SQLE and tumor progression in $\mathrm{BC}$, including DCIS lesions. In the present study, mRNA expression levels of SQLE in frozen DCIS and BC tissues and their adjacent normal breast tissues were assessed using RT-qPCR. The present data revealed that SQLE mRNA expression was significantly increased in DCIS and BC tissues compared with in adjacent normal tissues. This result demonstrated the oncogenic properties of SQLE in BC as well as in DCIS. However, there were no significant differences in SQLE mRNA expression between DCIS and BC. In accordance with the RT-qPCR findings, immunohistochemical analysis revealed that SQLE expression in DCIS and BC was significantly higher than that in normal breast tissues, but there was no significant difference in SQLE expression between DCIS and BC tissues. These results suggest that SQLE may serve an essential role in $\mathrm{BC}$ progression and may be especially activated during the process of DCIS development.

Although the underlying mechanism of SQLE dysregulation in $\mathrm{BC}$ remains unclear, SQLE is frequently altered by copy number gains in BC, and SQLE expression appears to be tightly regulated by increases in the copy dosage of its gene locus $(17,24,26)$. SQLE methylation has also been associated with SQLE overexpression in BC (25).

Recently, it has been suggested that the dysregulation of SQLE may be associated with the prognosis in patients with BC. Helms et al (17) compared SQLE mRNA expression of $\mathrm{BC}$ cases with and without chromosomal $7 \mathrm{p}$ and $8 \mathrm{q}$ gains by suppression subtractive hybridization PCR. SQLE mRNA expression was upregulated in $\mathrm{BC}$ with $\mathrm{ER}^{+} 7 \mathrm{p} / 8 \mathrm{q}$ gains (17). Although SQLE expression was not associated with tumor size, grade and ER or HER2 status, SQLE mRNA expression was an independent risk factor for the early onset of distant metastasis among early stage I/II BC cases (17). Simigdala et al (20) found that the cholesterol biosynthesis pathway was the common adaptive mechanism associated with acquired resistance to aromatase inhibitors in the ER+ long-term estrogen-deprived cell lines. The aforementioned study analyzed in-silico data from primary patients with $\mathrm{ER}+\mathrm{BC}$ with neoadjuvant aromatase inhibitor therapy and revealed that increased on-treatment SQLE expression was significantly associated with poor response to endocrine therapy (20). Kim et al (21) found that SQLE was one of the important genes for BC metastasis based on a standardized pathway-based approach. Shkurnikov et al (22) searched for novel parameters predicting the risk of relapse in patients with BC using public microarray datasets, revealing that SQLE expression was significantly associated with the risk of relapse in patients with $\mathrm{BC}$. Parada et al (23) examined racial differences in the expression levels of eight genes, including SQLE, and their associations with the risk of $\mathrm{BC}$ recurrence among white and black women with BC. Compared with white women, black women exhibited higher expression levels of SQLE, and high SQLE expression was associated with increased risk of BC recurrence (23). Chin et al (24) performed a high-resolution comparative genomic hybridization analysis in $\mathrm{BC}$, revealing that $8 \mathrm{q} 24$ locus, where the
SQLE gene resides, is one of the hotspot genomic regions exhibiting the strongest association between copy number gain and aberrant gene expression in high-grade, ER BC. Furthermore, amplification of 8q24 was associated with a poor prognosis independently of standard prognostic factors (24). Brown et al (26) independently confirmed that SQLE is $a$ bona fide oncogene by amplification with clinical relevance in BC. SQLE overexpression was more prevalent in high-grade, $\mathrm{HER}^{+}$and hormone receptor-negative invasive $\mathrm{BC}$, and was an independently significant unfavorable prognostic biomarker in BC (26). Yu et al (18) evaluated gene expression profiles from early-onset BC tissues (age of patients, $<40$ years) and their adjacent normal tissues to explore the genes and prognostic factors associated with $\mathrm{BC}$, revealing that SQLE expression was upregulated in BC and that high SQLE expression was associated with a poor prognosis.

Most studies on SQLE in BC have been based on molecular approaches. The present study investigated whether SQLE detection by immunohistochemistry could predict the prognosis in patients with BC. High SQLE expression was more prevalent in aggressive $\mathrm{BC}$, such as larger tumor size, nodal metastases, higher stage, HER2 subtype and distant metastatic relapse. High SQLE expression was associated with poor disease-free survival and overall survival, and independently predicted unfavorable disease-free survival in patients with BC. SQLE was subsequently verified as a prognostic biomarker for BC using the public BreastMark database. High SQLE mRNA expression was significantly associated with a poor prognosis in the 'all', lymph node negative, lymph node positive, luminal A subtype and luminal B subtype groups. The present results support the findings of previous studies $(17,18,20-24,26)$ and suggest that high SQLE expression assessed by immunohistochemistry may be associated with a more aggressive phenotype in $\mathrm{BC}$ and may be used as a prognostic marker in patients with BC.

In the present study, high SQLE expression in DCIS was significantly associated with high nuclear grade, comedo-type necrosis and HER2 positivity, which are risk factors for DCIS recurrence or progression (27). However, high SQLE expression was not associated with the recurrence of DCIS. Considering the potential prognostic value of SQLE expression in patients with DCIS, further studies in a large cohort of DCIS cases with long-term follow-up period are required.

Several studies have demonstrated that SQLE promotes cancer cell proliferation and migration, and the presence of SQLE inhibitors in both in vitro and in vivo models causes cancer cell death $(11,13)$. Brown et al $(26)$ demonstrated that SQLE inhibition decreased the viability of a BC cell line in a copy-dosage-dependent way and increased the doubling time only in BC cell lines with high SQLE expression. In the present study, SQLE mRNA expression was significantly increased in $\mathrm{BC}$ tissues compared with in adjacent normal breast tissues. The current findings may pave the way for the development of novel therapeutic strategies aimed at SQLE in BC. Further studies in vivo, such as animal models of $\mathrm{BC}$, are required to elucidate the mechanism of action of SQLE in promoting BC and to evaluate the therapeutic efficacy of SQLE inhibitors for the treatment of $\mathrm{BC}$.

The selection of appropriate internal control genes is crucial for proper interpretation of RT-qPCR data. In the present 
study, only the GAPDH gene was used as an internal reference control because the mRNA expression levels were constant in different tissue samples. It is recommended to use at least two reference genes to increase the resolution and accuracy of the RT-qPCR analysis (37). Therefore, the accuracy of the RT-qPCR results in the present study may be limited.

In summary, the current results suggested that upregulation of SQLE expression serves an important role in BC progression. Analysis of SQLE expression by immunohistochemistry may be a useful biomarker to predict the prognosis in patients with BC. Therefore, the present findings may open the way for further research in clinical settings to assess the relevance of SQLE inhibition as a new treatment option in patients with $\mathrm{BC}$.

\section{Acknowledgements}

Not applicable.

\section{Funding}

The present study was supported by a grant from the Chonnam National University Hwasun Hospital Institute for Biomedical Science (grant no. HCRI20013).

\section{Availability of data and materials}

The datasets generated and/or analyzed during the current study are not publicly available due to privacy and other restrictions, but are available from the corresponding author on reasonable request.

\section{Authors' contributions}

NIK conceived the experiments and wrote the manuscript. NC designed the experiments. MHP prepared the samples. NIK and MHP performed the experiments and confirmed the authenticity of all the raw data. NIK and NC processed and analyzed the data. SSK designed the study, performed statistical analysis and edited the manuscript. JSL developed the project, collected and analyzed the data, and wrote and revised the manuscript with input from all authors. All authors read and approved the final manuscript.

\section{Ethics approval and consent to participate}

The present study was a retrospective study that utilized archived materials of normal, DCIS and BC tissues and did not impact patient care; hence, approval was granted by the Institutional Review Board of Chonnam National University Hwasun Hospital (Jeollanam, Republic of Korea) without patient consent (reference no. CNUHH-2020-056).

\section{Patient consent for publication}

Not applicable.

\section{Competing interests}

The authors declare that they have no competing interests.

\section{References}

1. Park EH, Min SY, Kim Z, Yoon CS, Jung KW, Nam SJ, Oh SJ, Lee S, Park BW, Lim W, et al; Korean Breast Cancer Society: Basic facts of breast cancer in Korea in 2014: The 10-year overall survival progress. J Breast Cancer 20: 1-11, 2017.

2. Akram M, Iqbal M, Daniyal M and Khan AU: Awareness and current knowledge of breast cancer. Biol Res 50: 33, 2017.

3. Cirmena G, Franceschelli P, Isnaldi E, Ferrando L, De Mariano M, Ballestrero A and Zoppoli G: Squalene epoxidase as a promising metabolic target in cancer treatment. Cancer Lett 425: 13-20, 2018.

4. Silvente-Poirot S and Poirot M: Cholesterol metabolism and cancer: The good, the bad and the ugly. Curr Opin Pharmacol 12: 673-676, 2012.

5. Hu J, Locasale JW, Bielas JH, O'Sullivan J, Sheahan K, Cantley LC, Vander Heiden MG and Vitkup D: Heterogeneity of tumor-induced gene expression changes in the human metabolic network. Nat Biotechnol 31: 522-529, 2013.

6. Silvente-Poirot S and Poirot M: Cancer. Cholesterol and cancer, in the balance. Science 343: 1445-1446, 2014.

7. Silvente-Poirot S and Poirot M: Cholesterol epoxide hydrolase and cancer. Curr Opin Pharmacol 12: 696-703, 2012.

8. Nagai M, Sakakibara J, Wakui K, Fukushima Y, Igarashi S, Tsuji S, Arakawa M and Ono T; Nagai M1: Sakakibara J, Wakui K, Fukushima Y, Igarashi S, Tsuji S, Arakawa M and Ono T: Localization of the squalene epoxidase gene (SQLE) to human chromosome region 8q24.1. Genomics 44: 141-143, 1997.

9. Stopsack KH, Gerke TA, Sinnott JA, Penney KL, Tyekucheva S, Sesso HD, Andersson SO, Andrén O, Cerhan JR, Giovannucci EL, et al: Cholesterol metabolism and prostate cancer lethality. Cancer Res 76: 4785-4790, 2016.

10. Stopsack KH, Gerke TA, Andrén O, Andersson SO, Giovannucci EL, Mucci LA and Rider JR: Cholesterol uptake and regulation in high-grade and lethal prostate cancers. Carcinogenesis 38: 806-811, 2017.

11. Liu D, Wong CC, Fu L, Chen H, Zhao L, Li C, Zhou Y, Zhang Y, $\mathrm{Xu} \mathrm{W}$, Yang Y, et al: Squalene epoxidase drives NAFLD-induced hepatocellular carcinoma and is a pharmaceutical target. Sci Transl Med 10: 437, 2018.

12. Souchek JJ, Baine MJ, Lin C, Rachagani S, Gupta S, Kaur S, Lester K, Zheng D, Chen S, Smith L, et al: Unbiased analysis of pancreatic cancer radiation resistance reveals cholesterol biosynthesis as a novel target for radiosensitisation. Br J Cancer 111: 1139-1149, 2014.

13. Qin Y, Zhang Y, Tang Q, Jin L and Chen Y: SQLE induces epithelial-to-mesenchymal transition by regulating of miR-133b in esophageal squamous cell carcinoma. Acta Biochim Biophys Sin (Shanghai) 49: 138-148, 2017.

14. Zhang HY, Li HM, Yu Z, Yu XY and Guo K: Expression and significance of squalene epoxidase in squamous lung cancerous tissues and pericarcinoma tissues. Thorac Cancer 5: 275-280, 2014.

15. Römer AM, Lühr I, Klein A, Friedl A, Sebens S, Rösel F, Arnold N, Strauss A, Jonat W and Bauer M: Normal mammary fibroblasts induce reversion of the malignant phenotype in human primary breast cancer. Anticancer Res 33: 1525-1536, 2013.

16. Polycarpou-Schwarz M, Gross M, Mestdagh P, Schott J, Grund SE, Hildenbrand C, Rom J, Aulmann S, Sinn HP, Vandesompele $\mathrm{J}$, et al: The cancer-associated microprotein CASIMO1 controls cell proliferation and interacts with squalene epoxidase modulating lipid droplet formation. Oncogene 37: 4750-4768, 2018.

17. Helms MW, Kemming D, Pospisil H, Vogt U, Buerger H, Korsching E, Liedtke C, Schlotter CM, Wang A, Chan SY, et al: Squalene epoxidase, located on chromosome 8q24.1, is upregulated in $8 \mathrm{q}+$ breast cancer and indicates poor clinical outcome in stage I and II disease. Br J Cancer 99: 774-780, 2008.

18. Yu Z, He Q and $\mathrm{Xu} \mathrm{G}$ : Screening of prognostic factors in early-onset breast cancer. Technol Cancer Res Treat: Feb 7, 2020 (Epub ahead of print). doi: 10.1177/1533033819893670.

19. D'Arcy M, Fleming J, Robinson WR, Kirk EL, Perou CM and Troester MA: Race-associated biological differences among Luminal A breast tumors. Breast Cancer Res Treat 152: 437-448, 2015.

20. Simigdala N, Gao Q, Pancholi S, Roberg-Larsen H, Zvelebil M, Ribas R, Folkerd E, Thompson A, Bhamra A, Dowsett M, et al: Cholesterol biosynthesis pathway as a novel mechanism of resistance to estrogen deprivation in estrogen receptor-positive breast cancer. Breast Cancer Res 18: 58, 2016. 
21. Kim S, Kon M and DeLisi C: Pathway-based classification of cancer subtypes. Biol Direct 7: 21, 2012.

22. Shkurnikov MY, Galatenko VV, Lebedev AE, Podol'skii VE, Tonevitskii EA and Mal'tseva DV: On statistical relationship between ADRA2A expression and the risk of breast cancer relapse. Bull Exp Biol Med 157: 454-458, 2014.

23. Parada H Jr, Sun X, Fleming JM, Williams-DeVane CR, Kirk EL, Olsson LT, Perou CM, Olshan AF and Troester MA: Race-associated biological differences among luminal A and basal-like breast cancers in the Carolina Breast Cancer Study. Breast Cancer Res 19: 131, 2017.

24. Chin SF, Teschendorff AE, Marioni JC, Wang Y, Barbosa-Morais NL, Thorne NP, Costa JL, Pinder SE, van de Wiel MA, Green AR, et al: High-resolution aCGH and expression profiling identifies a novel genomic subtype of ER negative breast cancer. Genome Biol 8: R215, 2007.

25. Parris TZ, Kovács A, Hajizadeh S, Nemes S, Semaan M, Levin M, Karlsson P and Helou K: Frequent MYC coamplification and DNA hypomethylation of multiple genes on $8 \mathrm{q}$ in 8p11-p12-amplified breast carcinomas. Oncogenesis 3: e95, 2014

26. Brown DN, Caffa I, Cirmena G, Piras D, Garuti A, Gallo M, Alberti S, Nencioni A, Ballestrero A and Zoppoli G: Squalene epoxidase is a bona fide oncogene by amplification with clinical relevance in breast cancer. Sci Rep 6: 19435, 2016

27. Schnitt SJ, Allred C, Britton P, Ellis IO, Lakhani SR, Morrow M, Palazzo J, Reynolds C, Rutgers E, Simpson J, et al: Ductal carcinoma in situ. In: WHO Classification of Tumours of the Breast. Lakhani SR, Ellis IO, Tan PH and van de Vijver MJ (eds). IARC Press, Lyon, pp90-94, 2012

28. Kim GE, Kim NI, Lee JS, Park MH and Kang K: Differentially expressed genes in matched normal, cancer, and lymph node metastases predict clinical outcomes in patients with breast cancer. Appl Immunohistochem Mol Morphol 28: 111-122, 2020.

29. Madden SF, Clarke C, Gaule P, Aherne ST, O'Donovan N, Clynes M, Crown J and Gallagher WM: BreastMark: An integrated approach to mining publicly available transcriptomic datasets relating to breast cancer outcome. Breast Cancer Res 15: R52, 2013.

30. Xu YF, Yi Y, Qiu SJ, Gao Q, Li YW, Dai CX, Cai MY, Ju MJ, Zhou J, Zhang BH, et al: PEBP1 downregulation is associated to poor prognosis in $\mathrm{HCC}$ related to hepatitis B infection. $\mathrm{J}$ Hepatol 53: 872-879, 2010.
31. Livak KJ and Schmittgen TD: Analysis of relative gene expression data using real-time quantitative PCR and the 2(- $\Delta \Delta \mathrm{C}(\mathrm{T}))$ method. Methods 25: 402-408, 2001.

32. Hammond ME, Hayes DF, Dowsett M, Allred DC, Hagerty KL, Badve S, Fitzgibbons PL, Francis G, Goldstein NS, Hayes M, et al: American Society of Clinical Oncology/College of American Pathologists guideline recommendations for immunohistochemical testing of estrogen and progesterone receptors in breast cancer. Arch Pathol Lab Med 134: 907-922, 2010.

33. Wolff AC, Hammond ME, Schwartz JN, Hagerty KL, Allred DC, Cote RJ, Dowsett M, Fitzgibbons PL, Hanna WM, Langer A, et al; American Society of Clinical Oncology; College of American Pathologists: American Society of Clinical Oncology/College of American Pathologists guideline recommendations for human epidermal growth factor receptor 2 testing in breast cancer. J Clin Oncol 25: 118-145, 2007.

34. Bae YK, Gong G, Kang J, Lee A, Cho EY, Lee JS, Suh KS, Lee DW and Jung WH; Breast Pathology Study Group of Korean Society of Pathologists: HER2 status by standardized immunohistochemistry and silver-enhanced in situ hybridization in Korean breast cancer. J Breast Cancer 15: 381-387, 2012.

35. Kim GE, Kim JH, Lee KH, Choi YD, Lee JS, Lee JH, Nam JH, Choi C, Park MH and Yoon JH: Stromal matrix metalloproteinase-14 expression correlates with the grade and biological behavior of mammary phyllodes tumors. Appl Immunohistochem Mol Morphol 20: 298-303, 2012.

36. Jardel P, Debiais C, Godet J, Irani J and Fromont G: Ductal carcinoma of the prostate shows a different immunophenotype from high grade acinar cancer. Histopathology 63: 57-63, 2013.

37. Kozera B and Rapacz M: Reference genes in real-time PCR. J Appl Genet 54: 391-406, 2013.

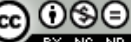

This work is licensed under a Creative Commons Attribution-NonCommercial-NoDerivatives 4.0 International (CC BY-NC-ND 4.0) License. 